\title{
Global dynamics of two systems of exponential difference equations by Lyapunov function
}

\section{Abdul Qadeer Khan*}

"Correspondence:

abdulqadeerkhan1@gmail.com Department of Mathematics,

University of Azad Jammu and

Kashmir, Muzaffarabad, Pakistan

\begin{abstract}
In this paper, we study the boundedness character and persistence, existence and uniqueness of the positive equilibrium, local and global behavior, and rate of convergence of positive solutions of two systems of exponential difference equations. Furthermore, by constructing a discrete Lyapunov function, we obtain the global asymptotic stability of the unique positive equilibrium point. Some numerical examples are given to verify our theoretical results.
\end{abstract}

MSC: 39A10; 40A05

Keywords: difference equations; boundedness; persistence; asymptotic behavior; Lyapunov function; rate of convergence

\section{Introduction and preliminaries}

Since difference equations and systems of difference equations containing exponential terms have many potential applications in biology, there are many papers dealing with such equations. See, for example the following.

El-Metwally et al. [1] have investigated the boundedness character, asymptotic behavior, periodicity nature of the positive solutions, and stability of the equilibrium point of the following population model:

$$
x_{n+1}=\alpha+\beta x_{n-1} e^{-x_{n}},
$$

where the parameters $\alpha, \beta$ are positive numbers and the initial conditions are arbitrary non-negative real numbers.

Ozturk et al. [2] have investigated the boundedness, asymptotic behavior, periodicity, and stability of the positive solutions of the following difference equation:

$$
y_{n+1}=\frac{\alpha+\beta e^{-y_{n}}}{\gamma+y_{n-1}},
$$

where the parameters $\alpha, \beta, \gamma$ are positive numbers and the initial conditions are arbitrary non-negative numbers.

\section{勧 Springer}

(อ2014 Khan; licensee Springer. This is an Open Access article distributed under the terms of the Creative Commons Attribution License (http://creativecommons.org/licenses/by/2.0), which permits unrestricted use, distribution, and reproduction in any medium, provided the original work is properly cited. 
Bozkurt [3] has investigated the local and global behavior of positive solutions of the following difference equation:

$$
y_{n+1}=\frac{\alpha e^{-y_{n}}+\beta e^{-y_{n-1}}}{\gamma+\alpha y_{n}+\beta y_{n-1}},
$$

where the parameters $\alpha, \beta, \gamma$, and the initial conditions are arbitrary positive numbers.

Papaschinopoulos et al. [4] have investigated the boundedness, persistence, and asymptotic behavior of positive solutions of the following two directional interactive and invasive species models:

$$
x_{n+1}=a+b x_{n-1} e^{-y_{n}}, \quad y_{n+1}=c+d y_{n-1} e^{-x_{n}},
$$

where the parameters $a, b, c, d$ and the initial conditions are arbitrary positive numbers.

Papaschinopoulos et al. [5] have investigated the asymptotic behavior of the solutions of the following three systems of difference equations of exponential form:

$$
\begin{aligned}
x_{n+1} & =\frac{\alpha+\beta e^{-y_{n}}}{\gamma+y_{n-1}}, & y_{n+1} & =\frac{\delta+\epsilon e^{-y_{n}}}{\zeta+x_{n-1}}, \\
x_{n+1} & =\frac{\alpha+\beta e^{-y_{n}}}{\gamma+x_{n-1}}, & y_{n+1} & =\frac{\delta+\epsilon e^{-x_{n}}}{\zeta+y_{n-1}}, \\
x_{n+1} & =\frac{\alpha+\beta e^{-x_{n}}}{\gamma+y_{n-1}}, & y_{n+1} & =\frac{\delta+\epsilon e^{-y_{n}}}{\zeta+x_{n-1}},
\end{aligned}
$$

where the parameters $\alpha, \beta, \gamma, \delta, \epsilon, \delta$ are positive numbers and the initial conditions are arbitrary non-negative numbers.

Papaschinopoulos and Schinas [6] have investigated the asymptotic behavior of the positive solutions of the systems of the two difference equations:

$$
\begin{array}{ll}
x_{n+1}=a+b y_{n-1} e^{-y_{n}}, & y_{n+1}=c+d x_{n-1} e^{-x_{n}}, \\
x_{n+1}=a+b y_{n-1} e^{-x_{n}}, & y_{n+1}=c+d x_{n-1} e^{-y_{n},},
\end{array}
$$

where the parameters $a, b, c, d$, and the initial conditions are arbitrary positive numbers.

Recently, Khan and Qureshi [7] have investigated the qualitative behavior of the following exponential type system of rational difference equations:

$$
x_{n+1}=\frac{\alpha e^{-y_{n}}+\beta e^{-y_{n-1}}}{\gamma+\alpha x_{n}+\beta x_{n-1}}, \quad y_{n+1}=\frac{\alpha_{1} e^{-x_{n}}+\beta_{1} e^{-x_{n-1}}}{\gamma_{1}+\alpha_{1} y_{n}+\beta_{1} y_{n-1}}, \quad n=0,1, \ldots
$$

where $\alpha, \beta, \gamma, \alpha_{1}, \beta_{1}, \gamma_{1}$, and the initial conditions $x_{0}, x_{-1}, y_{0}, y_{-1}$ are positive real numbers.

Motivated by the above studies, our aim in this paper is to investigate the qualitative behavior of positive solutions of the following two systems of exponential rational difference equations:

$$
x_{n+1}=\frac{\alpha e^{-y_{n}}+\beta e^{-y_{n-1}}}{\gamma+\alpha y_{n}+\beta y_{n-1}}, \quad y_{n+1}=\frac{\alpha_{1} e^{-x_{n}}+\beta_{1} e^{-x_{n-1}}}{\gamma_{1}+\alpha_{1} x_{n}+\beta_{1} x_{n-1}}, \quad n=0,1, \ldots,
$$


and

$$
x_{n+1}=\frac{\alpha e^{-x_{n}}+\beta e^{-x_{n-1}}}{\gamma+\alpha y_{n}+\beta y_{n-1}}, \quad y_{n+1}=\frac{\alpha_{1} e^{-y_{n}}+\beta_{1} e^{-y_{n-1}}}{\gamma_{1}+\alpha_{1} x_{n}+\beta_{1} x_{n-1}}, \quad n=0,1, \ldots
$$

where the parameters $\alpha, \beta, \gamma, \alpha_{1}, \beta_{1}, \gamma_{1}$, and the initial conditions are positive real numbers.

More precisely, we investigate the boundedness character, persistence, existence and uniqueness of positive steady state, local asymptotic stability and global behavior of the unique positive equilibrium point, and rate of convergence of positive solutions of systems (1) and (2) which converge to its unique positive equilibrium point. For basic theory and applications of difference equations we refer the reader [8-16] and references therein.

Let us consider the four-dimensional discrete dynamical system of the form

$$
x_{n+1}=f\left(x_{n}, x_{n-1}, y_{n}, y_{n-1}\right), \quad y_{n+1}=g\left(x_{n}, x_{n-1}, y_{n}, y_{n-1}\right), \quad n=0,1, \ldots,
$$

where $f: I^{2} \times J^{2} \rightarrow I$ and $g: I^{2} \times J^{2} \rightarrow J$ are continuously differentiable functions and $I, J$ are some intervals of real numbers. Furthermore, a solution $\left\{\left(x_{n}, y_{n}\right)\right\}_{n=-1}^{\infty}$ of system (3) is uniquely determined by the initial conditions $\left(x_{i}, y_{i}\right) \in I \times J$ for $i \in\{-1,0\}$. Along with system (3) we consider the corresponding vector map $F=\left(f, x_{n}, g, y_{n}\right)$. An equilibrium point of $(3)$ is a point $(\bar{x}, \bar{y})$ that satisfies

$$
\bar{x}=f(\bar{x}, \bar{x}, \bar{y}, \bar{y}), \quad \bar{y}=g(\bar{x}, \bar{x}, \bar{y}, \bar{y}) .
$$

The point $(\bar{x}, \bar{y})$ is also called a fixed point of the vector map $F$.

Definition 1 Let $(\bar{x}, \bar{y})$ be an equilibrium point of system (3).

(i) An equilibrium point $(\bar{x}, \bar{y})$ is said to be stable if, for every $\varepsilon>0$, there exists $\delta>0$ such that for every initial condition $\left(x_{i}, y_{i}\right), i \in\{-1,0\},\left\|\sum_{i=-1}^{0}\left(x_{i}, y_{i}\right)-(\bar{x}, \bar{y})\right\|<\delta$ implies $\left\|\left(x_{n}, y_{n}\right)-(\bar{x}, \bar{y})\right\|<\varepsilon$ for all $n>0$, where $\|\cdot\|$ is the usual Euclidean norm in $\mathbb{R}^{2}$.

(ii) An equilibrium point $(\bar{x}, \bar{y})$ is said to be unstable if it is not stable.

(iii) An equilibrium point $(\bar{x}, \bar{y})$ is said to be asymptotically stable if there exists $\eta>0$ such that $\left\|\sum_{i=-1}^{0}\left(x_{i}, y_{i}\right)-(\bar{x}, \bar{y})\right\|<\eta$ and $\left(x_{n}, y_{n}\right) \rightarrow(\bar{x}, \bar{y})$ as $n \rightarrow \infty$.

(iv) An equilibrium point $(\bar{x}, \bar{y})$ is called a global attractor if $\left(x_{n}, y_{n}\right) \rightarrow(\bar{x}, \bar{y})$ as $n \rightarrow \infty$.

(v) An equilibrium point $(\bar{x}, \bar{y})$ is called an asymptotic global attractor if it is a global attractor and stable.

Definition 2 Let $(\bar{x}, \bar{y})$ be an equilibrium point of the map

$$
F=\left(f, x_{n}, g, y_{n}\right) \text {, }
$$

where $f$ and $g$ are continuously differentiable functions at $(\bar{x}, \bar{y})$. The linearized system of (3) about the equilibrium point $(\bar{x}, \bar{y})$ is

$$
X_{n+1}=F\left(X_{n}\right)=F_{J} X_{n},
$$


where

$$
X_{n}=\left(\begin{array}{c}
x_{n} \\
x_{n-1} \\
y_{n} \\
y_{n-1}
\end{array}\right)
$$

and $F_{J}$ is the Jacobian matrix of system (3) about the equilibrium point $(\bar{x}, \bar{y})$.

Lemma 1 [17] Consider the system $X_{n+1}=F\left(X_{n}\right), n=0,1, \ldots$, where $\bar{X}$ is a fixed point of $F$. If all eigenvalues of the Jacobian matrix $J_{F}$ about $\bar{X}$ lie inside the open unit disk $|\lambda|<1$, then $\bar{X}$ is locally asymptotically stable. If any of the eigenvalue has a modulus greater than one, then $\bar{X}$ is unstable.

The following result gives the rate of convergence of solutions of a system of difference equations:

$$
X_{n+1}=(A+B(n)) X_{n},
$$

where $X_{n}$ is an $m$-dimensional vector, $A \in C^{m \times m}$ is a constant matrix, and $B: \mathbb{Z}^{+} \rightarrow C^{m \times m}$ is a matrix function satisfying

$$
\|B(n)\| \rightarrow 0
$$

as $n \rightarrow \infty$, where $\|\cdot\|$ denotes any matrix norm which is associated with the vector norm

$$
\|(x, y)\|=\sqrt{x^{2}+y^{2}}
$$

Proposition 1 (Perron's theorem) [18] Suppose that condition (5) holds. If $X_{n}$ is a solution of (4), then either $X_{n}=0$ for all large $n$ or

$$
\rho=\lim _{n \rightarrow \infty}\left(\left\|X_{n}\right\|\right)^{1 / n}
$$

or

$$
\rho=\lim _{n \rightarrow \infty} \frac{\left\|X_{n+1}\right\|}{\left\|X_{n}\right\|}
$$

exists and is equal to the modulus of one of the eigenvalues of matrix $A$.

2 On the system $x_{n+1}=\frac{\alpha e^{-y_{n}}+\beta e^{-y_{n-1}}}{\gamma+\alpha y_{n}+\beta y_{n-1}}, y_{n+1}=\frac{\alpha_{1} e^{-x_{n}}+\beta_{1} e^{-x_{n-1}}}{\gamma_{1}+\alpha_{1} x_{n}+\beta_{1} x_{n-1}}$

In this section, we shall investigate the asymptotic behavior of system (1). Let $(\bar{x}, \bar{y})$ be the equilibrium point of system (1) then

$$
\bar{x}=\frac{(\alpha+\beta) e^{-\bar{y}}}{\gamma+(\alpha+\beta) \bar{y}}, \quad \bar{y}=\frac{\left(\alpha_{1}+\beta_{1}\right) e^{-\bar{x}}}{\gamma_{1}+\left(\alpha_{1}+\beta_{1}\right) \bar{x}} .
$$

To construct the corresponding linearized form of system (1), we consider the following transformation:

$$
\left(x_{n}, x_{n-1}, y_{n}, y_{n-1}\right) \mapsto\left(f, f_{1}, g, g_{1}\right),
$$


where

$$
f=\frac{\alpha e^{-y_{n}}+\beta e^{-y_{n-1}}}{\gamma+\alpha y_{n}+\beta y_{n-1}}, \quad f_{1}=x_{n}, \quad g=\frac{\alpha_{1} e^{-x_{n}}+\beta_{1} e^{-x_{n-1}}}{\gamma_{1}+\alpha_{1} x_{n}+\beta_{1} x_{n-1}}, \quad g_{1}=y_{n} .
$$

The Jacobian matrix about the fixed point $(\bar{x}, \bar{y})$ under the transformation (6) is given by

$$
F_{J}(\bar{x}, \bar{y})=\left(\begin{array}{cccc}
0 & 0 & -\frac{\alpha\left(e^{-\bar{y}}+\bar{x}\right) \bar{y}}{\gamma+(\alpha+\beta) \bar{y}} & -\frac{\beta\left(e^{-\bar{y}}+\bar{x}\right)}{\gamma+(\alpha+\beta) \bar{y}} \\
1 & 0 & 0 & 0 \\
-\frac{\alpha_{1}\left(e^{-\bar{x}}+\bar{y}\right)}{\gamma_{1}+\left(\alpha_{1}+\beta_{1}\right) \bar{x}} & -\frac{\left.\beta_{1}\left(e^{-\bar{x}}+\bar{y}\right)\right)}{\gamma_{1}+\left(\alpha_{1}+\beta_{1}\right) \bar{x}} & 0 & 0 \\
0 & 0 & 1 & 0
\end{array}\right) .
$$

\subsection{Boundedness and persistence}

The following theorem shows that every positive solution $\left\{\left(x_{n}, y_{n}\right)\right\}$ of system (1) is bounded and persists.

Theorem 1 Every positive solution $\left\{\left(x_{n}, y_{n}\right)\right\}$ of system (1) is bounded and persists.

Proof Let $\left\{\left(x_{n}, y_{n}\right)\right\}$ be an arbitrary solution of (1). From (1), we have

$$
x_{n} \leq \frac{\alpha+\beta}{\gamma}=U_{1}, \quad y_{n} \leq \frac{\alpha_{1}+\beta_{1}}{\gamma_{1}}=U_{2}, \quad n=0,1,2, \ldots
$$

In addition from (1) and (7), we have

$$
x_{n} \geq \frac{(\alpha+\beta) e^{-\frac{\alpha_{1}+\beta_{1}}{\gamma_{1}}}}{\gamma+(\alpha+\beta) \frac{\alpha_{1}+\beta_{1}}{\gamma_{1}}}=L_{1}, \quad y_{n} \geq \frac{\left(\alpha_{1}+\beta_{1}\right) e^{-\frac{\alpha+\beta}{\gamma}}}{\gamma_{1}+\left(\alpha_{1}+\beta_{1}\right) \frac{\alpha+\beta}{\gamma}}=L_{2}, \quad n=2,3, \ldots .
$$

Hence, from (7) and (8), we get

$$
L_{1} \leq x_{n} \leq U_{1}, \quad L_{2} \leq y_{n} \leq U_{2}, \quad n=3,4, \ldots
$$

So the proof is complete.

\subsection{Existence of invariant set for solutions}

Theorem 2 Let $\left\{\left(x_{n}, y_{n}\right)\right\}$ be a positive solution of system (1). Then $\left[L_{1}, U_{1}\right] \times\left[L_{2}, U_{2}\right]$ is an invariant set for system (1).

Proof For any positive solution $\left\{\left(x_{n}, y_{n}\right)\right\}$ of system (1) with initial conditions $x_{0}, x_{-1} \in$ $\left[L_{1}, U_{1}\right]$, and $y_{0}, y_{-1} \in\left[L_{2}, U_{2}\right]$, we have

$$
x_{1}=\frac{\alpha e^{-y_{0}}+\beta e^{-y_{-1}}}{\gamma+\alpha y_{0}+\beta y_{-1}} \leq \frac{\alpha+\beta}{\gamma}
$$

and

$$
x_{1}=\frac{\alpha e^{-y_{0}}+\beta e^{-y_{-1}}}{\gamma+\alpha y_{0}+\beta y_{-1}} \geq \frac{(\alpha+\beta) e^{-\frac{\alpha_{1}+\beta_{1}}{\gamma_{1}}}}{\gamma+(\alpha+\beta) \frac{\alpha_{1}+\beta_{1}}{\gamma_{1}}} .
$$


Moreover,

$$
y_{1}=\frac{\alpha_{1} e^{-x_{0}}+\beta_{1} e^{-x_{-1}}}{\gamma_{1}+\alpha_{1} x_{0}+\beta_{1} x_{-1}} \leq \frac{\alpha_{1}+\beta_{1}}{\gamma_{1}}
$$

and

$$
y_{1}=\frac{\alpha_{1} e^{-x_{0}}+\beta_{1} e^{-x_{-1}}}{\gamma_{1}+\alpha_{1} x_{0}+\beta_{1} x_{-1}} \geq \frac{\left(\alpha_{1}+\beta_{1}\right) e^{-\frac{\alpha+\beta}{\gamma}}}{\gamma_{1}+\left(\alpha_{1}+\beta_{1}\right) \frac{\alpha+\beta}{\gamma}} .
$$

Hence, $x_{1} \in\left[L_{1}, U_{1}\right]$ and $y_{1} \in\left[L_{2}, U_{2}\right]$. Similarly, one can show that if $x_{k} \in\left[L_{1}, U_{1}\right]$ and $y_{k} \in\left[L_{2}, U_{2}\right]$, then $x_{k+1} \in\left[L_{1}, U_{1}\right]$ and $y_{k+1} \in\left[L_{2}, U_{2}\right]$.

\subsection{Existence and uniqueness of the positive equilibrium and local stability}

Theorem 3 Suppose that

$$
\eta<\left(\gamma\left(\gamma_{1}+\left(\alpha_{1}+\beta_{1}\right) L_{1}\right)+(\alpha+\beta)\left(\alpha_{1}+\beta_{1}\right) e^{-L_{1}}\right)^{2}\left(\gamma_{1}+\left(\alpha_{1}+\beta_{1}\right) L_{1}\right)
$$

where

$$
\begin{aligned}
\eta= & (\alpha+\beta)\left(\alpha_{1}+\beta_{1}\right) e^{-\frac{\left(\alpha_{1}+\beta_{1}\right) e^{-L_{1}}}{\gamma_{1}+\left(\alpha_{1}+\beta_{1}\right) L_{1}}-L_{1}}\left((\gamma+\alpha+\beta)\left(\gamma_{1}+\left(\alpha_{1}+\beta_{1}\right) U_{1}\right)\right. \\
& \left.+(\alpha+\beta)\left(\alpha_{1}+\beta_{1}\right) e^{-L_{1}}\right)\left(\gamma_{1}+\left(1+U_{1}\right)\left(\alpha_{1}+\beta_{1}\right)\right) .
\end{aligned}
$$

Then system (1) has a unique positive equilibrium point $(\bar{x}, \bar{y})$ in $\left[L_{1}, U_{1}\right] \times\left[L_{2}, U_{2}\right]$.

Proof Consider the following system of equations:

$$
x=\frac{(\alpha+\beta) e^{-y}}{\gamma+(\alpha+\beta) y}, \quad y=\frac{\left(\alpha_{1}+\beta_{1}\right) e^{-x}}{\gamma_{1}+\left(\alpha_{1}+\beta_{1}\right) x} .
$$

Let $F(x)=\frac{(\alpha+\beta) e^{-f(x)}}{\gamma+(\alpha+\beta) f(x)}-x$, where $f(x)=\frac{\left(\alpha_{1}+\beta_{1}\right) e^{-x}}{\gamma_{1}+\left(\alpha_{1}+\beta_{1}\right) x}$ and $x \in\left[L_{1}, U_{1}\right]$. Then it follows that $F\left(L_{1}\right)=\frac{(\alpha+\beta) e^{-f\left(L_{1}\right)}}{\gamma+(\alpha+\beta) f\left(L_{1}\right)}-L_{1}$. Now, $F\left(L_{1}\right)>0$ if and only if

$$
(\alpha+\beta) e^{-\frac{\left(\alpha_{1}+\beta_{1}\right) e^{-L_{1}}}{\gamma_{1}+\left(\alpha_{1}+\beta_{1}\right) L_{1}}}>L_{1}\left(\gamma+\frac{(\alpha+\beta)\left(\alpha_{1}+\beta_{1}\right) e^{-L_{1}}}{\gamma_{1}+\left(\alpha_{1}+\beta_{1}\right) L_{1}}\right) .
$$

Furthermore, we have $F\left(U_{1}\right)=\frac{(\alpha+\beta) e^{-f\left(U_{1}\right)}}{\gamma+(\alpha+\beta) f\left(U_{1}\right)}-U_{1}$ where $f\left(U_{1}\right)=\frac{\left(\alpha_{1}+\beta_{1}\right) e^{-U_{1}}}{\gamma_{1}+\left(\alpha_{1}+\beta_{1}\right) U_{1}}$. It is easy to see that $F\left(U_{1}\right)<0$ if and only if

$$
(\alpha+\beta) e^{-\frac{\left(\alpha_{1}+\beta_{1}\right) e^{-U_{1}}}{\gamma_{1}+\left(\alpha_{1}+\beta_{1}\right) U_{1}}}<U_{1}\left(\gamma+\frac{(\alpha+\beta)\left(\alpha_{1}+\beta_{1}\right) e^{-U_{1}}}{\gamma_{1}+\left(\alpha_{1}+\beta_{1}\right) U_{1}}\right) .
$$

Hence, $F(x)$ has at least one positive solution in $\left[L_{1}, U_{1}\right]$. Furthermore, assume that condition (9) is satisfied, then one has

$$
\frac{d F(x)}{d x}<-1+\frac{\eta}{\left(\gamma\left(\gamma_{1}+\left(\alpha_{1}+\beta_{1}\right) L_{1}\right)+(\alpha+\beta)\left(\alpha_{1}+\beta_{1}\right) e^{-L_{1}}\right)^{2}\left(\gamma_{1}+\left(\alpha_{1}+\beta_{1}\right) L_{1}\right)}<0 .
$$

Hence, $F(x)=0$ has a unique positive solution in $\left[L_{1}, U_{1}\right]$. This completes the proof. 
Theorem 4 Assume that

$$
(\alpha+\beta)\left(\alpha_{1}+\beta_{1}\right)\left(e^{-L_{2}}+U_{1}\right)\left(e^{-L_{1}}+U_{2}\right)<\left(\gamma+(\alpha+\beta) L_{2}\right)\left(\gamma_{1}+\left(\alpha_{1}+\beta_{1}\right) L_{1}\right) .
$$

Then the unique positive equilibrium point $(\bar{x}, \bar{y})$ in $\left[L_{1}, U_{1}\right] \times\left[L_{2}, U_{2}\right]$ of system $(1)$ is locally asymptotically stable.

Proof The characteristic polynomial of the Jacobian matrix $F_{J}(\bar{x}, \bar{y})$ about the equilibrium point $(\bar{x}, \bar{y})$ is given by

$$
\begin{aligned}
P(\lambda)= & \lambda^{4}-\frac{\alpha \alpha_{1}\left(e^{-\bar{y}}+\bar{x}\right)\left(e^{-\bar{x}}+\bar{y}\right)}{(\gamma+(\alpha+\beta) \bar{y})\left(\gamma_{1}+\left(\alpha_{1}+\beta_{1}\right) \bar{x}\right)} \lambda^{2}-\frac{\left(\alpha \beta_{1}+\beta \alpha_{1}\right)\left(e^{-\bar{y}}+\bar{x}\right)\left(e^{-\bar{x}}+\bar{y}\right)}{(\gamma+(\alpha+\beta) \bar{y})\left(\gamma_{1}+\left(\alpha_{1}+\beta_{1}\right) \bar{x}\right)} \lambda \\
& -\frac{\beta \beta_{1}\left(e^{-\bar{y}}+\bar{x}\right)\left(e^{-\bar{x}}+\bar{y}\right)}{(\gamma+(\alpha+\beta) \bar{y})\left(\gamma_{1}+\left(\alpha_{1}+\beta_{1}\right) \bar{x}\right)} .
\end{aligned}
$$

Let $\Phi(\lambda)=\lambda^{2}$ and

$$
\begin{aligned}
\Psi(\lambda)= & \frac{\alpha \alpha_{1}\left(e^{-\bar{y}}+\bar{x}\right)\left(e^{-\bar{x}}+\bar{y}\right)}{(\gamma+(\alpha+\beta) \bar{y})\left(\gamma_{1}+\left(\alpha_{1}+\beta_{1}\right) \bar{x}\right)} \lambda^{2}+\frac{\left(\alpha \beta_{1}+\beta \alpha_{1}\right)\left(e^{-\bar{y}}+\bar{x}\right)\left(e^{-\bar{x}}+\bar{y}\right)}{(\gamma+(\alpha+\beta) \bar{y})\left(\gamma_{1}+\left(\alpha_{1}+\beta_{1}\right) \bar{x}\right)} \lambda \\
& +\frac{\beta \beta_{1}\left(e^{-\bar{y}}+\bar{x}\right)\left(e^{-\bar{x}}+\bar{y}\right)}{(\gamma+(\alpha+\beta) \bar{y})\left(\gamma_{1}+\left(\alpha_{1}+\beta_{1}\right) \bar{x}\right)} .
\end{aligned}
$$

Assume that $(\alpha+\beta)\left(\alpha_{1}+\beta_{1}\right)\left(e^{-L_{2}}+U_{1}\right)\left(e^{-L_{1}}+U_{2}\right)<\left(\gamma+(\alpha+\beta) L_{2}\right)\left(\gamma_{1}+\left(\alpha_{1}+\beta_{1}\right) L_{1}\right)$. Then one has

$$
\begin{aligned}
|\Psi(\lambda)| \leq & \frac{\alpha \alpha_{1}\left(e^{-\bar{y}}+\bar{x}\right)\left(e^{-\bar{x}}+\bar{y}\right)}{(\gamma+(\alpha+\beta) \bar{y})\left(\gamma_{1}+\left(\alpha_{1}+\beta_{1}\right) \bar{x}\right)}+\frac{\left(\alpha \beta_{1}+\beta \alpha_{1}\right)\left(e^{-\bar{y}}+\bar{x}\right)\left(e^{-\bar{x}}+\bar{y}\right)}{(\gamma+(\alpha+\beta) \bar{y})\left(\gamma_{1}+\left(\alpha_{1}+\beta_{1}\right) \bar{x}\right)} \\
& +\frac{\beta \beta_{1}\left(e^{-\bar{y}}+\bar{x}\right)\left(e^{-\bar{x}}+\bar{y}\right)}{(\gamma+(\alpha+\beta) \bar{y})\left(\gamma_{1}+\left(\alpha_{1}+\beta_{1}\right) \bar{x}\right)} \\
= & \left(\alpha \alpha_{1}+\alpha \beta_{1}+\beta \alpha_{1}+\beta \beta_{1}\right)\left(\frac{\left(e^{-\bar{y}}+\bar{x}\right)\left(e^{-\bar{x}}+\bar{y}\right)}{(\gamma+(\alpha+\beta) \bar{y})\left(\gamma_{1}+\left(\alpha_{1}+\beta_{1}\right) \bar{x}\right)}\right) \\
< & \frac{(\alpha+\beta)\left(\alpha_{1}+\beta_{1}\right)\left(e^{-L_{2}}+U_{1}\right)\left(e^{-L_{1}}+U_{2}\right)}{\left(\gamma+(\alpha+\beta) L_{2}\right)\left(\gamma_{1}+\left(\alpha_{1}+\beta_{1}\right) L_{1}\right)}<1 .
\end{aligned}
$$

Then, by Rouche's theorem, $\Phi(\lambda)$ and $\Phi(\lambda)-\Psi(\lambda)$ have the same number of zeroes in an open unit disk $|\lambda|<1$. Hence, the unique positive equilibrium point $(\bar{x}, \bar{y})$ in $\left[L_{1}, U_{1}\right] \times$ $\left[L_{2}, U_{2}\right]$ of system $(1)$ is locally asymptotically stable.

\subsection{Global character}

\section{Theorem 5 If}

$$
(\alpha+\beta) e^{-L_{2}}<\bar{x}\left(\gamma+(\alpha+\beta) L_{2}\right) \text { and }\left(\alpha_{1}+\beta_{1}\right) e^{-L_{1}}<\bar{y}\left(\gamma_{1}+\left(\alpha_{1}+\beta_{1}\right) L_{1}\right),
$$

then the unique positive equilibrium point $(\bar{x}, \bar{y})$ of system (1) is globally asymptotically stable.

Proof Arranging as in [19], we consider the following discrete time analog of the Lyapunov function: 


$$
V_{n}=\bar{x}\left(\frac{x_{n}}{\bar{x}}-1-\ln \frac{x_{n}}{\bar{x}}\right)+\bar{y}\left(\frac{y_{n}}{\bar{y}}-1-\ln \frac{y_{n}}{\bar{y}}\right) .
$$

The nonnegativity of $V_{n}$ follows from the following inequality:

$$
x-1-\ln x \geq 0, \quad \forall x>0 .
$$

Furthermore, we have

$$
\begin{aligned}
& -\ln \left(\frac{x_{n+1}}{x_{n}}\right)=\ln \left(1-\left(1-\frac{x_{n}}{x_{n+1}}\right)\right) \leq-\frac{x_{n+1}-x_{n}}{x_{n+1}}, \\
& -\ln \left(\frac{y_{n+1}}{y_{n}}\right)=\ln \left(1-\left(1-\frac{y_{n}}{y_{n+1}}\right)\right) \leq-\frac{y_{n+1}-y_{n}}{y_{n+1}} .
\end{aligned}
$$

Assume that (10) holds true, then it follows that

$$
\begin{aligned}
V_{n+1}-V_{n}= & \bar{x}\left(\frac{x_{n+1}}{\bar{x}}-1-\ln \frac{x_{n+1}}{\bar{x}}\right)+\bar{y}\left(\frac{y_{n+1}}{\bar{y}}-1-\ln \frac{y_{n+1}}{\bar{y}}\right)-\bar{x}\left(\frac{x_{n}}{\bar{x}}-1-\ln \frac{x_{n}}{\bar{x}}\right) \\
& -\bar{y}\left(\frac{y_{n}}{\bar{y}}-1-\ln \frac{y_{n}}{\bar{y}}\right) \\
\leq & \left(x_{n+1}-x_{n}\right)\left(1-\frac{\bar{x}}{x_{n+1}}\right)+\left(y_{n+1}-y_{n}\right)\left(1-\frac{\bar{y}}{y_{n+1}}\right) \\
= & \left(x_{n+1}-x_{n}\right)\left(\frac{\alpha e^{-y_{n}}+\beta e^{-y_{n-1}}-\bar{x}\left(\gamma+\alpha y_{n}+\beta y_{n-1}\right)}{\alpha e^{-y_{n}}+\beta e^{-y_{n-1}}}\right) \\
& +\left(y_{n+1}-y_{n}\right)\left(\frac{\alpha_{1} e^{-x_{n}}+\beta_{1} e^{-x_{n-1}}-\bar{y}\left(\gamma_{1}+\alpha_{1} x_{n}+\beta_{1} x_{n-1}\right)}{\alpha_{1} e^{-x_{n}}+\beta_{1} e^{-x_{n-1}}}\right) \\
\leq & \left(U_{1}-L_{1}\right)\left(\frac{(\alpha+\beta) e^{-L_{2}}-\bar{x}\left(\gamma+(\alpha+\beta) L_{2}\right)}{(\alpha+\beta) e^{-L_{2}}}\right) \\
& +\left(U_{2}-L_{2}\right)\left(\frac{\left(\alpha_{1}+\beta_{1}\right) e^{-L_{1}}-\bar{y}\left(\gamma_{1}+\left(\alpha_{1}+\beta_{1}\right) L_{1}\right)}{\left(\alpha_{1}+\beta_{1}\right) e^{-L_{1}}}\right) \leq 0,
\end{aligned}
$$

for all $n \geq 0$. Thus $V_{n}$ is a non-increasing non-negative sequence. It follows that $\lim _{n \rightarrow \infty} V_{n} \geq 0$. Hence, we obtain $\lim _{n \rightarrow \infty}\left(V_{n+1}-V_{n}\right)=0$. Then it follows that $\lim _{n \rightarrow \infty} x_{n+1}=\bar{x}$ and $\lim _{n \rightarrow \infty} y_{n+1}=\bar{y}$. Furthermore, $V_{n} \leq V_{0}$ for all $n \geq 0$, which shows that $(\bar{x}, \bar{y}) \in\left[L_{1}, U_{1}\right] \times\left[L_{2}, U_{2}\right]$ is uniformly stable. Hence, the unique positive equilibrium point $(\bar{x}, \bar{y}) \in\left[L_{1}, U_{1}\right] \times\left[L_{2}, U_{2}\right]$ of system $(1)$ is globally asymptotically stable.

\subsection{Rate of convergence}

In this section, we will determine the rate of convergence of a solution that converges to the unique positive equilibrium point of system (1).

Let $\left\{\left(x_{n}, y_{n}\right)\right\}$ be any solution of system (1) such that $\lim _{n \rightarrow \infty} x_{n}=\bar{x}$, and $\lim _{n \rightarrow \infty} y_{n}=\bar{y}$. To find the error terms, note that

$$
\begin{aligned}
x_{n+1}-\bar{x} & =\frac{\alpha e^{-y_{n}}+\beta e^{-y_{n-1}}}{\gamma+\alpha y_{n}+\beta y_{n-1}}-\frac{(\alpha+\beta) e^{-\bar{y}}}{\gamma+(\alpha+\beta) \bar{y}} \\
& =-\frac{\alpha e^{-y_{n}}\left(e^{y_{n}-\bar{y}}-1\right)}{\gamma+\alpha y_{n}+\beta y_{n-1}}-\frac{\beta e^{-y_{n-1}\left(e^{y_{n-1}-\bar{y}}-1\right)}}{\gamma+\alpha y_{n}+\beta y_{n-1}}
\end{aligned}
$$




$$
\begin{aligned}
& -\frac{\alpha \bar{x}\left(y_{n}-\bar{y}\right)}{\gamma+\alpha y_{n}+\beta y_{n-1}}-\frac{\beta \bar{x}\left(y_{n-1}-\bar{y}\right)}{\gamma+\alpha y_{n}+\beta y_{n-1}} \\
& =-\frac{\alpha e^{-y_{n}}\left(y_{n}-\bar{y}+O_{1}\left(\left(y_{n}-\bar{y}\right)^{2}\right)\right)}{\gamma+\alpha y_{n}+\beta y_{n-1}}-\frac{\beta e^{-y_{n-1}}\left(y_{n-1}-\bar{y}+O_{2}\left(\left(y_{n-1}-\bar{y}\right)^{2}\right)\right)}{\gamma+\alpha y_{n}+\beta y_{n-1}} \\
& -\frac{\alpha \bar{x}\left(y_{n}-\bar{y}\right)}{\gamma+\alpha y_{n}+\beta y_{n-1}}-\frac{\beta \bar{x}\left(y_{n-1}-\bar{y}\right)}{\gamma+\alpha y_{n}+\beta y_{n-1}} \text {. }
\end{aligned}
$$

So,

$$
\begin{aligned}
x_{n+1}-\bar{x}= & -\frac{\alpha\left(e^{-y_{n}}+\bar{x}\right)}{\gamma+\alpha y_{n}+\beta y_{n-1}}\left(y_{n}-\bar{y}\right)-\frac{\beta\left(e^{\left.-y_{n-1}+\bar{x}\right)}\right.}{\gamma+\alpha y_{n}+\beta y_{n-1}}\left(y_{n-1}-\bar{y}\right)+O_{1}\left(\left(y_{n}-\bar{y}\right)^{2}\right) \\
& +O_{2}\left(\left(y_{n-1}-\bar{y}\right)^{2}\right) .
\end{aligned}
$$

Similarly,

$$
\begin{aligned}
y_{n+1}-\bar{y}= & -\frac{\alpha_{1}\left(e^{-x_{n}}+\bar{y}\right)}{\gamma_{1}+\alpha_{1} x_{n}+\beta x_{n-1}}\left(x_{n}-\bar{x}\right)-\frac{\beta_{1}\left(e^{-x_{n-1}}+\bar{y}\right)}{\gamma_{1}+\alpha_{1} x_{n}+\beta_{1} x_{n-1}}\left(x_{n-1}-\bar{x}\right)+O_{3}\left(\left(x_{n}-\bar{x}\right)^{2}\right) \\
& +O_{4}\left(\left(x_{n-1}-\bar{x}\right)^{2}\right) .
\end{aligned}
$$

From (11) and (12), we have

$$
\begin{aligned}
& x_{n+1}-\bar{x} \approx-\frac{\alpha\left(e^{-y_{n}}+\bar{x}\right)}{\gamma+\alpha y_{n}+\beta y_{n-1}}\left(y_{n}-\bar{y}\right)-\frac{\beta\left(e^{-y_{n-1}}+\bar{x}\right)}{\gamma+\alpha y_{n}+\beta y_{n-1}}\left(y_{n-1}-\bar{y}\right), \\
& y_{n+1}-\bar{y} \approx-\frac{\alpha_{1}\left(e^{-x_{n}}+\bar{y}\right)}{\gamma_{1}+\alpha_{1} x_{n}+\beta x_{n-1}}\left(x_{n}-\bar{x}\right)-\frac{\beta_{1}\left(e^{-x_{n-1}}+\bar{y}\right)}{\gamma_{1}+\alpha_{1} x_{n}+\beta_{1} x_{n-1}}\left(x_{n-1}-\bar{x}\right) .
\end{aligned}
$$

Let $e_{n}^{1}=x_{n}-\bar{x}$ and $e_{n}^{2}=y_{n}-\bar{y}$. Then system (13) can be represented as

$$
e_{n+1}^{1} \approx a_{n} e_{n}^{2}+b_{n} e_{n-1}^{2}, \quad e_{n+1}^{2} \approx c_{n} e_{n}^{1}+d_{n} e_{n-1}^{1}
$$

where

$$
\begin{array}{ll}
a_{n}=-\frac{\alpha\left(e^{-y_{n}}+\bar{x}\right)}{\gamma+\alpha y_{n}+\beta y_{n-1}}, & b_{n}=-\frac{\beta\left(e^{-y_{n-1}}+\bar{x}\right)}{\gamma+\alpha y_{n}+\beta y_{n-1}}, \\
c_{n}=-\frac{\alpha_{1}\left(e^{-x_{n}}+\bar{y}\right)}{\gamma_{1}+\alpha_{1} x_{n}+\beta x_{n-1}}, & d_{n}=-\frac{\beta_{1}\left(e^{-x_{n-1}}+\bar{y}\right)}{\gamma_{1}+\alpha_{1} x_{n}+\beta_{1} x_{n-1}} .
\end{array}
$$

Moreover,

$$
\begin{array}{ll}
\lim _{n \rightarrow \infty} a_{n}=-\frac{\alpha\left(e^{-\bar{y}}+\bar{x}\right)}{\gamma+(\alpha+\beta) \bar{y}}, & \lim _{n \rightarrow \infty} b_{n}=-\frac{\beta\left(e^{-\bar{y}}+\bar{x}\right)}{\gamma+(\alpha+\beta) \bar{y}}, \\
\lim _{n \rightarrow \infty} c_{n}=-\frac{\alpha_{1}\left(e^{-\bar{x}}+\bar{y}\right)}{\gamma_{1}+\left(\alpha_{1}+\beta_{1}\right) \bar{x}}, & \lim _{n \rightarrow \infty} d_{n}=-\frac{\beta_{1}\left(e^{-\bar{x}}+\bar{y}\right)}{\gamma_{1}+\left(\alpha_{1}+\beta_{1}\right) \bar{x}} .
\end{array}
$$

So, the limiting system of the error terms can be written as

$$
\left(\begin{array}{c}
e_{n+1}^{1} \\
e_{n}^{1} \\
e_{n+1}^{2} \\
e_{n}^{2}
\end{array}\right)=\left(\begin{array}{cccc}
0 & 0 & -\frac{\alpha\left(e^{-\bar{y}}+\bar{x}\right)}{\gamma+(\alpha+\beta) \bar{y}} & -\frac{\beta\left(e^{-\bar{y}}+\bar{x}\right)}{\gamma+(\alpha+\beta) \bar{y}} \\
1 & 0 & 0 & 0 \\
-\frac{\alpha_{1}\left(e^{-\bar{x}}+\bar{y}\right)}{\gamma_{1}+\left(\alpha_{1}+\beta_{1}\right) \bar{x}} & -\frac{\beta_{1}\left(e^{-\bar{x}}+\bar{y}\right)}{\gamma_{1}+\left(\alpha_{1}+\beta_{1}\right) \bar{x}} & 0 & 0 \\
0 & 0 & 1 & 0
\end{array}\right)\left(\begin{array}{c}
e_{n}^{1} \\
e_{n-1}^{1} \\
e_{n}^{2} \\
e_{n-2}^{2}
\end{array}\right),
$$


which is similar to the linearized system of (1) about the equilibrium point $(\bar{x}, \bar{y})$. Using Proposition 1, one has the following result.

Theorem 6 Assume that $\left\{\left(x_{n}, y_{n}\right)\right\}$ be a positive solution of system (1) such that $\lim _{n \rightarrow \infty} x_{n}=$ $\bar{x}$, and $\lim _{n \rightarrow \infty} y_{n}=\bar{y}$, where $\bar{x}$ in $\left[L_{1}, U_{1}\right]$ and $\bar{y}$ in $\left[L_{2}, U_{2}\right]$. Then the error vector

$$
e_{n}=\left(\begin{array}{c}
e_{n}^{1} \\
e_{n-1}^{1} \\
e_{n}^{2} \\
e_{n-2}^{2}
\end{array}\right)
$$

of every solution of (1) satisfies both of the following asymptotic relations:

$$
\lim _{n \rightarrow \infty}\left(\left\|e_{n}\right\|\right)^{\frac{1}{n}}=\left|\lambda_{1,2,3,4} F_{J}(\bar{x}, \bar{y})\right|, \quad \lim _{n \rightarrow \infty} \frac{\left\|e_{n+1}\right\|}{\left\|e_{n}\right\|}=\left|\lambda_{1,2,3,4} F_{J}(\bar{x}, \bar{y})\right|,
$$

where $\lambda_{1,2,3,4} F_{J}(\bar{x}, \bar{y})$ are the characteristic roots of Jacobian matrix $F_{J}(\bar{x}, \bar{y})$.

3 On the system $x_{n+1}=\frac{\alpha e^{-x_{n}}+\beta e^{-x_{n-1}}}{\gamma+\alpha y_{n}+\beta y_{n-1}}, y_{n+1}=\frac{\alpha_{1} e^{-y_{n}}+\beta_{1} e^{-y_{n-1}}}{\gamma_{1}+\alpha_{1} x_{n}+\beta_{1} x_{n-1}}$

In this section, we shall investigate the asymptotic behavior of system (2). Let $(\bar{x}, \bar{y})$ be the equilibrium point of system (2), then

$$
\bar{x}=\frac{(\alpha+\beta) e^{-\bar{x}}}{\gamma+(\alpha+\beta) \bar{y}}, \quad \bar{y}=\frac{\left(\alpha_{1}+\beta_{1}\right) e^{-\bar{y}}}{\gamma_{1}+\left(\alpha_{1}+\beta_{1}\right) \bar{x}} .
$$

To construct the corresponding linearized form of system (2), we consider the following transformation:

$$
\left(x_{n}, x_{n-1}, y_{n}, y_{n-1}\right) \mapsto\left(f, f_{1}, g, g_{1}\right),
$$

where

$$
f=\frac{\alpha e^{-x_{n}}+\beta e^{-x_{n-1}}}{\gamma+\alpha y_{n}+\beta y_{n-1}}, \quad f_{1}=x_{n}, \quad g=\frac{\alpha_{1} e^{-y_{n}}+\beta_{1} e^{-y_{n-1}}}{\gamma_{1}+\alpha_{1} x_{n}+\beta_{1} x_{n-1}}, \quad g_{1}=y_{n} .
$$

The Jacobian matrix about the fixed point $(\bar{x}, \bar{y})$ under transformation (14) is given by

$$
F_{J}(\bar{x}, \bar{y})=\left(\begin{array}{cccc}
A & B & C & D \\
1 & 0 & 0 & 0 \\
A_{1} & B_{1} & C_{1} & D_{1} \\
0 & 0 & 1 & 0
\end{array}\right),
$$

where $A=-\frac{\alpha e^{-\bar{x}}}{\gamma+(\alpha+\beta) \bar{y}}, B=-\frac{\beta e^{-\bar{x}}}{\gamma+(\alpha+\beta) \bar{y}}, C=-\frac{\alpha \bar{x}}{\gamma+(\alpha+\beta) \bar{y}}, D=-\frac{\beta \bar{x}}{\gamma+(\alpha+\beta)}, A_{1}=-\frac{\alpha_{1} \bar{y}}{\gamma_{1}+\left(\alpha_{1}+\beta_{1}\right) \bar{x}}, B_{1}=$ $-\frac{\beta_{1} \bar{y}}{\gamma_{1}+\left(\alpha_{1}+\beta_{1}\right) \bar{x}}, C_{1}=-\frac{\alpha_{1} e^{-\bar{y}}}{\gamma_{1}+\left(\alpha_{1}+\beta_{1}\right) \bar{x}}, D_{1}=-\frac{\beta_{1} e^{-\bar{y}}}{\gamma_{1}+\left(\alpha_{1}+\beta_{1}\right)}$.

\subsection{Boundedness and persistence}

Theorem 7 Every positive solution $\left\{\left(x_{n}, y_{n}\right)\right\}$ of system (2) is bounded and persists. 
Proof Let $\left\{\left(x_{n}, y_{n}\right)\right\}$ be an arbitrary solution of (2), then

$$
x_{n} \leq \frac{\alpha+\beta}{\gamma}=U_{1}, \quad y_{n} \leq \frac{\alpha_{1}+\beta_{1}}{\gamma_{1}}=U_{2}, \quad n=0,1, \ldots
$$

From (2) and (15), we have

$$
x_{n} \geq \frac{(\alpha+\beta) e^{-\frac{\alpha+\beta}{\gamma}}}{\gamma+(\alpha+\beta) \frac{\alpha_{1}+\beta_{1}}{\gamma_{1}}}=L_{1}, \quad y_{n} \geq \frac{\left(\alpha_{1}+\beta_{1}\right) e^{-\frac{\alpha_{1}+\beta_{1}}{\gamma_{1}}}}{\gamma_{1}+\left(\alpha_{1}+\beta_{1}\right) \frac{\alpha+\beta}{\gamma}}=L_{2}, \quad n=2,3, \ldots .
$$

Hence, from (15) and (16), we get

$$
L_{1} \leq x_{n} \leq U_{1}, \quad L_{2} \leq y_{n} \leq U_{2}, \quad n=3,4, \ldots
$$

This proves the statement.

Theorem 8 Let $\left\{\left(x_{n}, y_{n}\right)\right\}$ be a positive solution of system (2). Then $\left[L_{1}, U_{1}\right] \times\left[L_{2}, U_{2}\right]$ is an invariant set for system (2).

Proof Follows by induction.

\subsection{Existence and uniqueness and local stability}

The following theorem shows the existence and uniqueness of the positive equilibrium point of system (2).

Theorem 9 If

$$
\left(U_{1}+1\right) e^{-\left(L_{1}+\frac{e^{-L_{1}}}{L_{1}}-\frac{\gamma}{\alpha+\beta}\right)}\left(\frac{e^{-L_{1}}}{L_{1}}-\frac{\gamma}{\alpha+\beta}+1\right)<L_{1}^{2}\left(\frac{e^{-L_{1}}}{L_{1}}-\frac{\gamma}{\alpha+\beta}\right)^{2}
$$

then system (2) has a unique positive equilibrium point $(\bar{x}, \bar{y})$ in $\left[L_{1}, U_{1}\right] \times\left[L_{2}, U_{2}\right]$.

Proof Consider the following system of algebraic equations:

$$
x=\frac{(\alpha+\beta) e^{-x}}{\gamma+(\alpha+\beta) y}, \quad y=\frac{\left(\alpha_{1}+\beta_{1}\right) e^{-y}}{\gamma_{1}+\left(\alpha_{1}+\beta_{1}\right) x} .
$$

Assume that $(x, y) \in\left[L_{1}, U_{1}\right] \times\left[L_{2}, U_{2}\right]$, then it follows from (18) that

$$
y=\frac{e^{-x}}{x}-\frac{\gamma}{\alpha+\beta}, \quad x=\frac{e^{-y}}{y}-\frac{\gamma_{1}}{\alpha_{1}+\beta_{1}} .
$$

Defining

$$
F(x)=\frac{e^{-h(x)}}{h(x)}-\frac{\gamma_{1}}{\alpha_{1}+\beta_{1}}-x,
$$

where $h(x)=\frac{e^{-x}}{x}-\frac{\gamma}{\alpha+\beta}, x \in\left[L_{1}, U_{1}\right]$. It is easy to see that $F\left(L_{1}\right)=\frac{e^{-h\left(L_{1}\right)}}{h\left(L_{1}\right)}-\frac{\gamma_{1}}{\alpha_{1}+\beta_{1}}-L_{1}>0$ if and only if $e^{-\left(\frac{e^{-L_{1}}}{L_{1}}-\frac{\gamma}{\alpha+\beta}\right)}>\left(\frac{e^{-L_{1}}}{L_{1}}-\frac{\gamma}{\alpha+\beta}\right)\left(L_{1}+\frac{\gamma_{1}}{\alpha_{1}+\beta_{1}}\right)$. Also, $F\left(U_{1}\right)=\frac{e^{-h\left(U_{1}\right)}}{h\left(U_{1}\right)}-\frac{\gamma_{1}}{\alpha_{1}+\beta_{1}}-U_{1}<0$ if and 
only if $e^{-\left(\frac{e^{-U_{1}}}{U_{1}}-\frac{\gamma}{\alpha+\beta}\right)}<\left(\frac{e^{-U_{1}}}{U_{1}}-\frac{\gamma}{\alpha+\beta}\right)\left(U_{1}+\frac{\gamma_{1}}{\alpha_{1}+\beta_{1}}\right)$. Hence, $F(x)$ has at least one positive solution in $\left[L_{1}, U_{1}\right]$. Furthermore, assume that condition (17) is satisfied, then one has

$$
\begin{aligned}
F^{\prime}(x) & =\frac{(x+1) e^{-\left(x+\frac{e^{-x}}{x}-\frac{\gamma}{\alpha+\beta}\right)}\left(\frac{e^{-x}}{x}-\frac{\gamma}{\alpha+\beta}+1\right)}{x^{2}\left(\frac{e^{-x}}{x}-\frac{\gamma}{\alpha+\beta}\right)^{2}}-1 \\
& \leq \frac{\left(U_{1}+1\right) e^{-\left(L_{1}+\frac{e^{-L_{1}}}{L_{1}}-\frac{\gamma}{\alpha+\beta}\right)}\left(\frac{e^{-L_{1}}}{L_{1}}-\frac{\gamma}{\alpha+\beta}+1\right)}{L_{1}^{2}\left(\frac{e^{-L_{1}}}{L_{1}}-\frac{\gamma}{\alpha+\beta}\right)^{2}}-1<0 .
\end{aligned}
$$

Hence, $F(x)=0$ has a unique positive solution in $\left[L_{1}, U_{1}\right]$. This completes the proof.

\section{Theorem 10 If}

$$
\begin{aligned}
(\alpha & +\beta)\left(\alpha_{1}+\beta_{1}\right)\left(e^{-L_{1}-L_{2}}+U_{1} U_{2}\right) \\
& <\left(1-U_{1}-U_{2}\right)\left(\gamma+(\alpha+\beta) L_{2}\right)\left(\gamma_{1}+\left(\alpha_{1}+\beta_{1}\right) L_{1}\right),
\end{aligned}
$$

then the unique positive equilibrium point $(\bar{x}, \bar{y})$ of system (2) is locally asymptotically stable.

Proof The characteristic equation of the Jacobian matrix $F_{J}(\bar{x}, \bar{y})$ about the equilibrium point $(\bar{x}, \bar{y})$ is given by

$$
\lambda^{4}-p_{4} \lambda^{3}+p_{3} \lambda^{2}+p_{2} \lambda+p_{1}=0
$$

where $p_{4}=A+C_{1}, p_{3}=A C_{1}-B-A_{1} C-D_{1}, p_{2}=A D_{1}-A_{1} D+B C_{1}-B_{1} C, p_{1}=B D_{1}-B_{1} D$. Assuming condition (19) one has

$$
\begin{aligned}
\sum_{i=1}^{4}\left|p_{i}\right|= & \frac{(\alpha+\beta) e^{-\bar{x}}}{\gamma+(\alpha+\beta) \bar{y}}+\frac{\left(\alpha_{1}+\beta_{1}\right) e^{-\bar{y}}}{\gamma_{1}+\left(\alpha_{1}+\beta_{1}\right) \bar{x}} \\
& +\frac{\left(\alpha \alpha_{1}+\alpha \beta_{1}+\alpha_{1} \beta+\beta \beta_{1}\right) e^{-\bar{x}-\bar{y}}+\left(\alpha \alpha_{1}+\alpha \beta_{1}+\alpha_{1} \beta+\beta \beta_{1}\right) \bar{x} \bar{y}}{(\gamma+(\alpha+\beta) \bar{y})\left(\gamma_{1}+\left(\alpha_{1}+\beta_{1}\right) \bar{x}\right)} \\
= & \bar{x}+\bar{y}+\frac{(\alpha+\beta)\left(\alpha_{1}+\beta_{1}\right)\left(e^{-\bar{x}-\bar{y}}+\bar{x} \bar{y}\right)}{(\gamma+(\alpha+\beta) \bar{y})\left(\gamma_{1}+\left(\alpha_{1}+\beta_{1}\right) \bar{x}\right)} \\
< & U_{1}+U_{2}+\frac{(\alpha+\beta)\left(\alpha_{1}+\beta_{1}\right)\left(e^{-L_{1}-L_{2}}+U_{1} U_{2}\right)}{\left(\gamma+(\alpha+\beta) L_{2}\right)\left(\gamma_{1}+\left(\alpha_{1}+\beta_{1}\right) L_{1}\right)}<1 .
\end{aligned}
$$

Therefore, inequality (20) and Remark 1.3.1 of reference [20] implies that the unique positive equilibrium point $(\bar{x}, \bar{y})$ of system (2) is locally asymptotically stable. This completes the proof.

\subsection{Global character}

Theorem 11 If

$$
(\alpha+\beta) e^{-L_{1}}<\bar{x}\left(\gamma+(\alpha+\beta) L_{2}\right) \text { and }\left(\alpha_{1}+\beta_{1}\right) e^{-L_{2}}<\bar{y}\left(\gamma_{1}+\left(\alpha_{1}+\beta_{1}\right) L_{1}\right),
$$

then the unique positive equilibrium point $(\bar{x}, \bar{y})$ of system (2) is globally asymptotically stable. 
Proof Using arrangements for the proof of Theorem 5 and assume that (21) holds true, then

$$
\begin{aligned}
V_{n+1}-V_{n} \leq & \left(U_{1}-L_{1}\right)\left(\frac{(\alpha+\beta) e^{-L_{1}}-\bar{x}\left(\gamma+(\alpha+\beta) L_{2}\right)}{(\alpha+\beta) e^{-L_{1}}}\right) \\
& +\left(U_{2}-L_{2}\right)\left(\frac{\left(\alpha_{1}+\beta_{1}\right) e^{-L_{2}}-\bar{y}\left(\gamma_{1}+\left(\alpha_{1}+\beta_{1}\right) L_{1}\right)}{\left(\alpha_{1}+\beta_{1}\right) e^{-L_{2}}}\right) \leq 0
\end{aligned}
$$

for all $n \geq 0$ so that $V_{n} \geq 0$ is a non-increasing sequence. It follows that $\lim _{n \rightarrow \infty} V_{n} \geq 0$. Hence, we obtain $\lim _{n \rightarrow \infty}\left(V_{n+1}-V_{n}\right)=0$. It follows that $\lim _{n \rightarrow \infty} x_{n+1}=\bar{x}$ and $\lim _{n \rightarrow \infty} y_{n+1}=$ $\bar{y}$. Furthermore, $V_{n} \leq V_{0}$ for all $n \geq 0$, which implies that $(\bar{x}, \bar{y}) \in\left[L_{1}, U_{1}\right] \times\left[L_{2}, U_{2}\right]$ is uniform stable. Hence, the unique positive equilibrium point $(\bar{x}, \bar{y}) \in\left[L_{1}, U_{1}\right] \times\left[L_{2}, U_{2}\right]$ of system (2) is globally asymptotically stable.

\subsection{Rate of convergence}

In this section we will determine the rate of convergence of a solution that converges to the unique positive equilibrium point of system (2).

Let $\left\{\left(x_{n}, y_{n}\right)\right\}$ be any solution of system (2) such that $\lim _{n \rightarrow \infty} x_{n}=\bar{x}$, and $\lim _{n \rightarrow \infty} y_{n}=\bar{y}$. To find the error terms,

$$
\begin{aligned}
x_{n+1}-\bar{x}= & \frac{\alpha e^{-x_{n}}+\beta e^{-x_{n-1}}}{\gamma+\alpha y_{n}+\beta y_{n-1}}-\frac{(\alpha+\beta) e^{-\bar{x}}}{\gamma+(\alpha+\beta) \bar{y}} \\
= & -\frac{\alpha e^{-x_{n}}\left(e^{x_{n}-\bar{x}}-1\right)}{\gamma+\alpha y_{n}+\beta y_{n-1}}-\frac{\beta e^{-x_{n-1}\left(e^{x_{n-1}-\bar{x}}-1\right)}}{\gamma+\alpha y_{n}+\beta y_{n-1}}-\frac{\alpha \bar{x}\left(y_{n}-\bar{y}\right)}{\gamma+\alpha y_{n}+\beta y_{n-1}}-\frac{\beta \bar{x}\left(y_{n-1}-\bar{y}\right)}{\gamma+\alpha y_{n}+\beta y_{n-1}} \\
= & -\frac{\alpha e^{-x_{n}}\left(x_{n}-\bar{x}+O_{1}\left(\left(x_{n}-\bar{x}\right)^{2}\right)\right)}{\gamma+\alpha y_{n}+\beta y_{n-1}}-\frac{\beta e^{-x_{n-1}}\left(x_{n-1}-\bar{x}+O_{2}\left(\left(x_{n-1}-\bar{x}\right)^{2}\right)\right)}{\gamma+\alpha y_{n}+\beta y_{n-1}} \\
& -\frac{\alpha \bar{x}\left(y_{n}-\bar{y}\right)}{\gamma+\alpha y_{n}+\beta y_{n-1}}-\frac{\beta \bar{x}\left(y_{n-1}-\bar{y}\right)}{\gamma+\alpha y_{n}+\beta y_{n-1}} .
\end{aligned}
$$

So,

$$
\begin{aligned}
x_{n+1}-\bar{x}= & -\frac{\alpha e^{-x_{n}}}{\gamma+\alpha y_{n}+\beta y_{n-1}}\left(x_{n}-\bar{x}\right)-\frac{\beta e^{-x_{n-1}}}{\gamma+\alpha y_{n}+\beta y_{n-1}}\left(x_{n-1}-\bar{x}\right) \\
& -\frac{\alpha \bar{x}}{\gamma+\alpha y_{n}+\beta y_{n-1}}\left(y_{n}-\bar{y}\right)-\frac{\beta \bar{x}}{\gamma+\alpha y_{n}+\beta y_{n-1}}\left(y_{n-1}-\bar{y}\right) \\
& +O_{1}\left(\left(x_{n}-\bar{x}\right)^{2}\right)+O_{2}\left(\left(x_{n-1}-\bar{x}\right)^{2}\right) .
\end{aligned}
$$

Similarly,

$$
\begin{aligned}
y_{n+1}-\bar{y}= & -\frac{\alpha_{1} \bar{y}}{\gamma_{1}+\alpha_{1} x_{n}+\beta_{1} x_{n-1}}\left(x_{n}-\bar{x}\right)-\frac{\beta_{1} \bar{y}}{\gamma_{1}+\alpha_{1} x_{n}+\beta_{1} x_{n-1}}\left(x_{n-1}-\bar{x}\right) \\
& -\frac{\alpha_{1} e^{-y_{n}}}{\gamma_{1}+\alpha_{1} x_{n}+\beta_{1} x_{n-1}}\left(y_{n}-\bar{y}\right)-\frac{\beta_{1} e^{-y_{n-1}}}{\gamma_{1}+\alpha_{1} x_{n}+\beta_{1} x_{n-1}}\left(y_{n-1}-\bar{y}\right) \\
& +O_{3}\left(\left(y_{n}-\bar{y}\right)^{2}\right)+O_{4}\left(\left(y_{n-1}-\bar{y}\right)^{2}\right) .
\end{aligned}
$$


From (22) and (23), we have

$$
\left.\begin{array}{r}
x_{n+1}-\bar{x} \approx-\frac{\alpha e^{-x_{n}}}{\gamma+\alpha y_{n}+\beta y_{n-1}}\left(x_{n}-\bar{x}\right)-\frac{\beta e^{-x_{n-1}}}{\gamma+\alpha y_{n}+\beta y_{n-1}}\left(x_{n-1}-\bar{x}\right) \\
\quad-\frac{\alpha \bar{x}}{\gamma+\alpha y_{n}+\beta y_{n-1}}\left(y_{n}-\bar{y}\right)-\frac{\beta \bar{x}}{\gamma+\alpha y_{n}+\beta y_{n-1}}\left(y_{n-1}-\bar{y}\right), \\
y_{n+1}-\bar{y} \approx-\frac{\alpha y_{1}}{\gamma_{1}+\alpha_{1} x_{n}+\beta_{1} x_{n-1}}\left(x_{n}-\bar{x}\right)-\frac{\beta_{1} \bar{y}}{\gamma_{1}+\alpha_{1} x_{n}+\beta_{1} x_{n-1}}\left(x_{n-1}-\bar{x}\right) \\
-\frac{\alpha_{1} e^{-y_{n}}}{\gamma_{1}+\alpha_{1} x_{n}+\beta_{1} x_{n-1}}\left(y_{n}-\bar{y}\right)-\frac{\beta_{1} e^{-y_{n-1}}}{\gamma_{1}+\alpha_{1} x_{n}+\beta_{1} x_{n-1}}\left(y_{n-1}-\bar{y}\right) .
\end{array}\right\}
$$

Let $e_{n}^{1}=x_{n}-\bar{x}$, and $e_{n}^{2}=y_{n}-\bar{y}$. Then system (24) can be represented as

$$
e_{n+1}^{1} \approx a_{n} e_{n}^{1}+b_{n} e_{n-1}^{1}+c_{n} e_{n}^{2}+d_{n} e_{n-1}^{2}, \quad e_{n+1}^{2} \approx e_{n} e_{n}^{1}+f_{n} e_{n-1}^{1}+g_{n} e_{n}^{2}+h_{n} e_{n-1}^{2},
$$

where

$$
\begin{array}{ll}
a_{n}=-\frac{\alpha e^{-x_{n}}}{\gamma+\alpha y_{n}+\beta y_{n-1}}, & b_{n}=-\frac{\beta e^{-x_{n-1}}}{\gamma+\alpha y_{n}+\beta y_{n-1}}, \quad c_{n}=-\frac{\alpha \bar{x}}{\gamma+\alpha y_{n}+\beta y_{n-1}}, \\
d_{n}=-\frac{\beta \bar{x}}{\gamma+\alpha y_{n}+\beta y_{n-1}}, & e_{n}=-\frac{\alpha_{1} \bar{y}}{\gamma_{1}+\alpha_{1} x_{n}+\beta_{1} x_{n-1}}, \quad f_{n}=-\frac{\beta_{1} \bar{y}}{\gamma_{1}+\alpha_{1} x_{n}+\beta_{1} x_{n-1}}, \\
g_{n}=-\frac{\alpha_{1} e^{-y_{n}}}{\gamma_{1}+\alpha_{1} x_{n}+\beta_{1} x_{n-1}}, & h_{n}=-\frac{\beta_{1} e^{-y_{n-1}}}{\gamma_{1}+\alpha_{1} x_{n}+\beta_{1} x_{n-1}} .
\end{array}
$$

Moreover,

$$
\begin{array}{ll}
\lim _{n \rightarrow \infty} a_{n}=-\frac{\alpha e^{-\bar{x}}}{\gamma+(\alpha+\beta) \bar{y}}, & \lim _{n \rightarrow \infty} b_{n}=-\frac{\beta e^{-\bar{x}}}{\gamma+(\alpha+\beta) \bar{y}}, \\
\lim _{n \rightarrow \infty} c_{n}=-\frac{\alpha \bar{x}}{\gamma+(\alpha+\beta) \bar{y}}, & \lim _{n \rightarrow \infty} d_{n}=-\frac{\beta \bar{x}}{\gamma+(\alpha+\beta) \bar{y}} . \\
\lim _{n \rightarrow \infty} e_{n}=-\frac{\alpha_{1} \bar{y}}{\gamma_{1}+\left(\alpha_{1}+\beta_{1}\right) \bar{x}}, & \lim _{n \rightarrow \infty} f_{n}=-\frac{\beta_{1} \bar{y}}{\gamma_{1}+\left(\alpha_{1}+\beta_{1}\right) \bar{x}}, \\
\lim _{n \rightarrow \infty} g_{n}=-\frac{\alpha_{1} e^{-\bar{y}}}{\gamma_{1}+\left(\alpha_{1}+\beta_{1}\right) \bar{x}}, & \lim _{n \rightarrow \infty} h_{n}=-\frac{\beta_{1} e^{-\bar{y}}}{\gamma_{1}+\left(\alpha_{1}+\beta_{1}\right) \bar{x}} .
\end{array}
$$

So, the limiting system of the error terms can be written as

$$
\left(\begin{array}{c}
e_{n+1}^{1} \\
e_{n}^{1} \\
e_{n+1}^{2} \\
e_{n}^{2}
\end{array}\right)=\left(\begin{array}{cccc}
-\frac{\alpha e^{-\bar{x}}}{\gamma+(\alpha+\beta) \bar{y}} & -\frac{\beta e^{-\bar{x}}}{\gamma+(\alpha+\beta) \bar{y}} & -\frac{\alpha \bar{x}}{\gamma+(\alpha+\beta) \bar{y}} & -\frac{\beta \bar{x}}{\gamma+(\alpha+\beta) \bar{y}} \\
1 & 0 & 0 & 0 \\
-\frac{\alpha_{1} \bar{y}}{\gamma_{1}+\left(\alpha_{1}+\beta_{1}\right) \bar{x}} & -\frac{\beta_{1} \bar{y}}{\gamma_{1}+\left(\alpha_{1}+\beta_{1}\right) \bar{x}} & -\frac{\alpha_{1} e^{-\bar{y}}}{\gamma_{1}+\left(\alpha_{1}+\beta_{1}\right) \bar{x}} & -\frac{\beta_{1} e^{-\bar{y}}}{\gamma_{1}+\left(\alpha_{1}+\beta_{1}\right) \bar{x}} \\
0 & 0 & 1 & 0
\end{array}\right)\left(\begin{array}{c}
e_{n}^{1} \\
e_{n-1}^{1} \\
e_{n}^{2} \\
e_{n-2}^{2}
\end{array}\right),
$$

which is similar to linearized system of (2) about the equilibrium point $(\bar{x}, \bar{y})$. Using Proposition 1 , one has the following result.

Theorem 12 Assume that $\left\{\left(x_{n}, y_{n}\right)\right\}$ is a positive solution of system (2) such that $\lim _{n \rightarrow \infty} x_{n}=\bar{x}$, and $\lim _{n \rightarrow \infty} y_{n}=\bar{y}$, where $\bar{x}$ in $\left[L_{1}, U_{1}\right]$ and $\bar{y}$ in $\left[L_{2}, U_{2}\right]$. Then the error vector

$$
e_{n}=\left(\begin{array}{c}
e_{n}^{1} \\
e_{n-1}^{1} \\
e_{n}^{2} \\
e_{n-2}^{2}
\end{array}\right)
$$


of every solution of (2) satisfies both of the following asymptotic relations:

$$
\lim _{n \rightarrow \infty}\left(\left\|e_{n}\right\|\right)^{\frac{1}{n}}=\left|\lambda_{1,2,3,4} F_{J}(\bar{x}, \bar{y})\right|, \quad \lim _{n \rightarrow \infty} \frac{\left\|e_{n+1}\right\|}{\left\|e_{n}\right\|}=\left|\lambda_{1,2,3,4} F_{J}(\bar{x}, \bar{y})\right|,
$$

where $\lambda_{1,2,3,4} F_{J}(\bar{x}, \bar{y})$ are the roots of the characteristic polynomial of $F_{J}(\bar{x}, \bar{y})$.

\section{Examples}

In order to verify our theoretical results and to support our theoretical discussions, we consider several interesting numerical examples. These examples represent different types of qualitative behavior of solutions of the systems of nonlinear difference equations (1) and (2). All plots in this section are drawn with Mathematica.

Example 1 Let $\alpha=0.0005, \beta=3,024, \gamma=1,128, \alpha_{1}=1,005, \beta_{1}=1,025, \gamma_{1}=1,022$. Then system (1) can be written as

$$
x_{n+1}=\frac{0.0005 e^{-y_{n}}+3,024 e^{-y_{n-1}}}{1,128+0.0005 y_{n}+3,024 y_{n-1}}, \quad y_{n+1}=\frac{1,005 e^{-x_{n}}+1,025 e^{-x_{n-1}}}{1,022+1,005 x_{n}+1,025 x_{n-1}},
$$

with initial conditions $x_{-1}=1.8, x_{0}=0.01, y_{-1}=110.9, y_{0}=1.8$.

In this case the unique positive equilibrium point of system $(25)$ is given by $(\bar{x}, \bar{y})=$ $(2.39624,0.0314033)$. Moreover, in Figure 1 the plot of $x_{n}$ is shown in Figure 1(a), the plot of $y_{n}$ is shown in Figure 1(b), and an attractor of system (25) is shown in Figure 1(c).

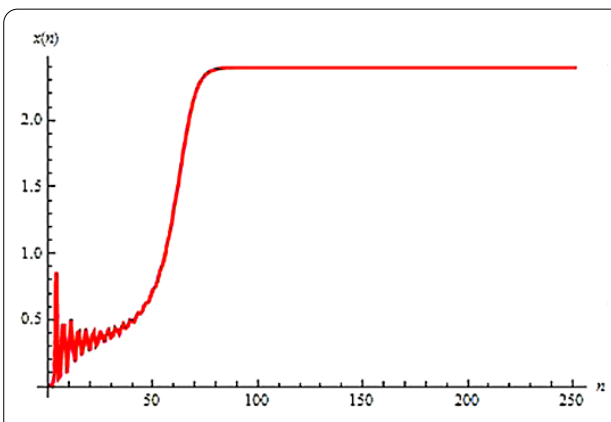

(a) Plot of $x_{n}$ for system (25)

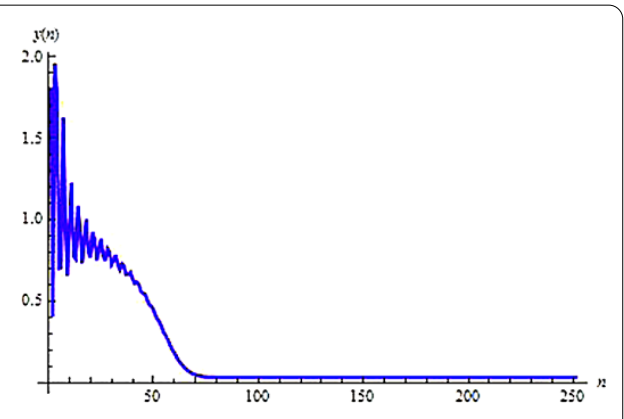

(b) Plot of $y_{n}$ for system (25)

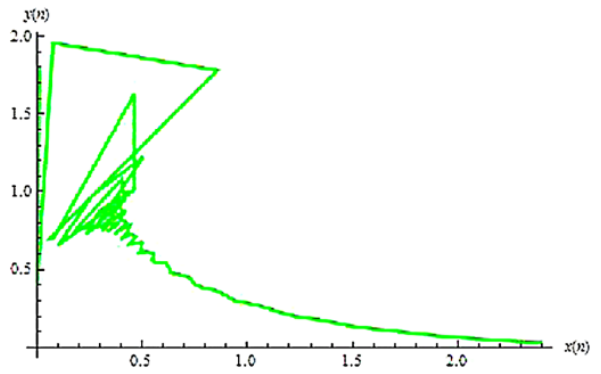

(c) An attractor of system (25)

Figure 1 Plots for system (25). 


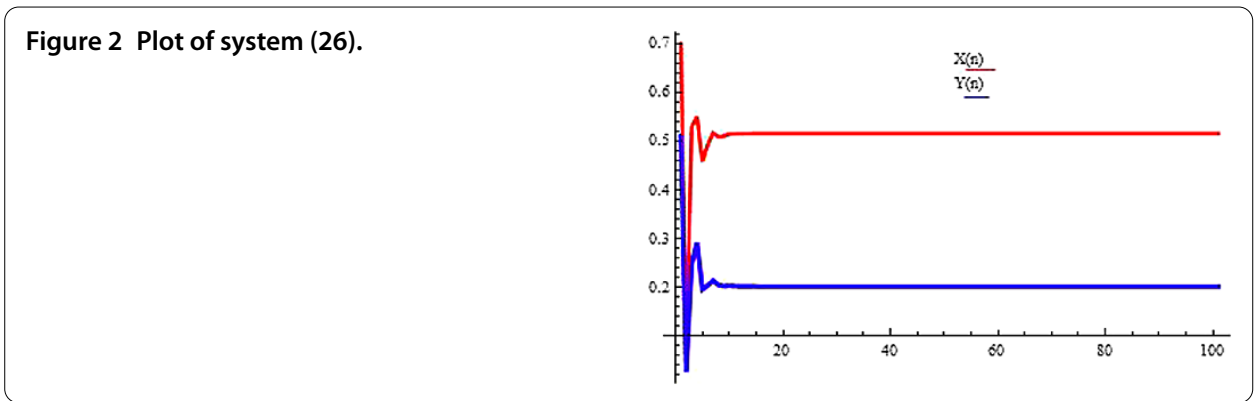

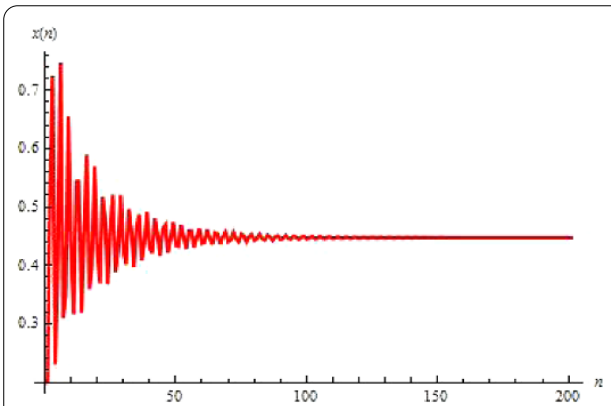

(a) Plot of $x_{n}$ for system (27)

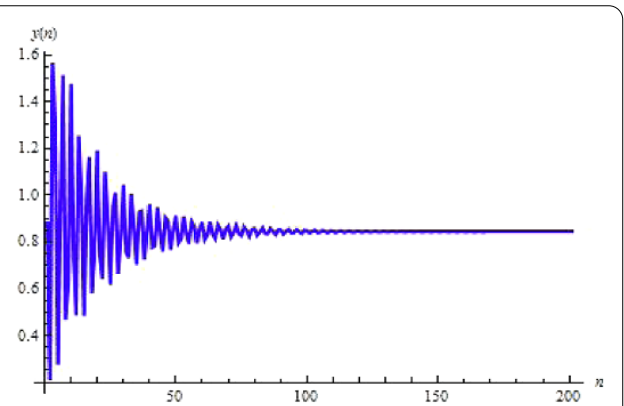

(b) Plot of $y_{n}$ for system (27)

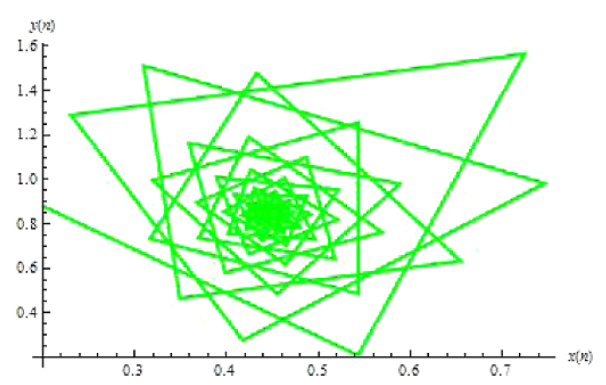

(c) An attractor of system (27)

Figure 3 Plots for system (27).

Example 2 Let $\alpha=230, \beta=132, \gamma=500, \alpha_{1}=111, \beta_{1}=135, \gamma_{1}=600$. Then system (1) can be written as

$$
x_{n+1}=\frac{230 e^{-y_{n}}+132 e^{-y_{n-1}}}{500+230 y_{n}+132 y_{n-1}}, \quad y_{n+1}=\frac{111 e^{-x_{n}}+135 e^{-x_{n-1}}}{600+111 x_{n}+135 x_{n-1}}, \quad n=0,1, \ldots
$$

with initial conditions $x_{-1}=10.9, x_{0}=0.7, y_{-1}=17.8, y_{0}=0.51$. The plot of system (26) is shown in Figure 2.

Example 3 Let $\alpha=50, \beta=1.4, \gamma=30, \alpha_{1}=0.5, \beta_{1}=12.5, \gamma_{1}=0.8$. Then system (2) can be written as

$$
x_{n+1}=\frac{50 e^{-x_{n}}+1.4 e^{-x_{n-1}}}{30+50 y_{n}+1.4 y_{n-1}}, \quad y_{n+1}=\frac{0.5 e^{-y_{n}}+12.5 e^{-y_{n-1}}}{0.8+0.5 x_{n}+12.5 x_{n-1}},
$$

with initial conditions $x_{-1}=0.8, x_{0}=0.2, y_{-1}=1.8, y_{0}=0.88$. 


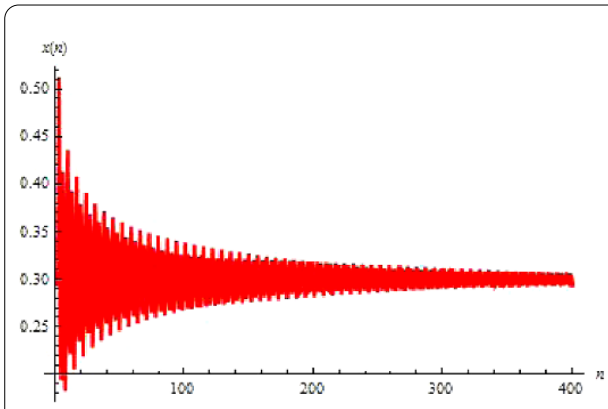

(a) Plot of $x_{n}$ for system (28)

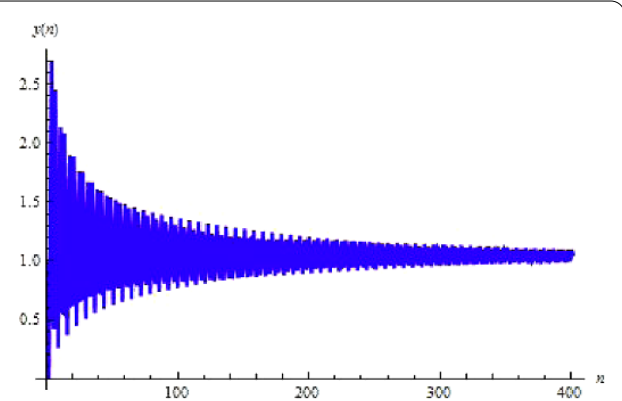

(b) Plot of $y_{n}$ for system (28)

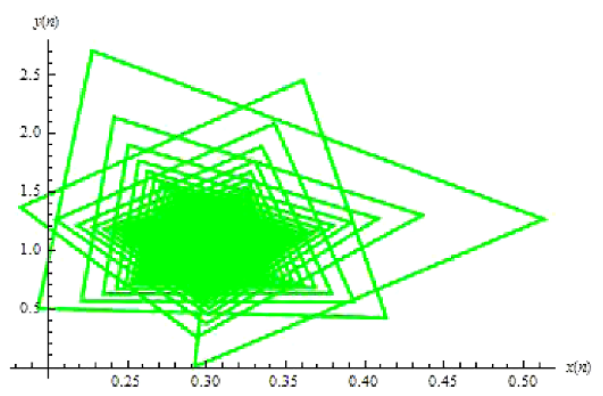

(c) An attractor of system (28)

Figure 4 Plots for system (28).

In this case the unique positive equilibrium point of system (27) is given by $(\bar{x}, \bar{y})=$ $(0.447606,0.844287)$. Moreover, in Figure 3 the plot of $x_{n}$ is shown in Figure 3(a), the plot of $y_{n}$ is shown in Figure 3(b), and an attractor of system (27) is shown in Figure 3(c).

Example 4 Let $\alpha=45, \beta=1.4, \gamma=66, \alpha_{1}=1.1, \beta_{1}=12.5, \gamma_{1}=0.5$. Then system (2) can be written as

$$
x_{n+1}=\frac{45 e^{-x_{n}}+1.4 e^{-x_{n-1}}}{66+45 y_{n}+1.4 y_{n-1}}, \quad y_{n+1}=\frac{1.1 e^{-y_{n}}+12.5 e^{-y_{n-1}}}{0.5+1.1 x_{n}+12.5 x_{n-1}},
$$

with initial conditions $x_{-1}=21.7, x_{0}=0.3, y_{-1}=2.8, y_{0}=0.98$.

In this case the unique positive equilibrium point of system (28) is given by $(\bar{x}, \bar{y})=$ $(0.300252,1.04429)$. Moreover, in Figure 4 the plot of $x_{n}$ is shown in Figure 4(a), the plot of $y_{n}$ is shown in Figure 4(b), and an attractor of system (28) is shown in Figure 4(c).

Example 5 Let $\alpha=125, \beta=4.5, \gamma=112, \alpha_{1}=18, \beta_{1}=32, \gamma_{1}=0.09$. Then system (2) can be written as

$$
x_{n+1}=\frac{125 e^{-x_{n}}+4.5 e^{-x_{n-1}}}{112+125 y_{n}+4.5 y_{n-1}}, \quad y_{n+1}=\frac{18 e^{-y_{n}}+32 e^{-y_{n-1}}}{0.09+18 x_{n}+32 x_{n-1}},
$$

with initial conditions $x_{-1}=20.7, x_{0}=0.3, y_{-1}=0.9, y_{0}=0.2$.

In this case the unique positive equilibrium point of system (29) is given by $(\bar{x}, \bar{y})=$ $(0.371312,0.992951)$. Moreover, in Figure 5 the plot of $x_{n}$ is shown in Figure 5(a), the plot of $y_{n}$ is shown in Figure 5(b), and an attractor of system (29) is shown in Figure 5(c). 


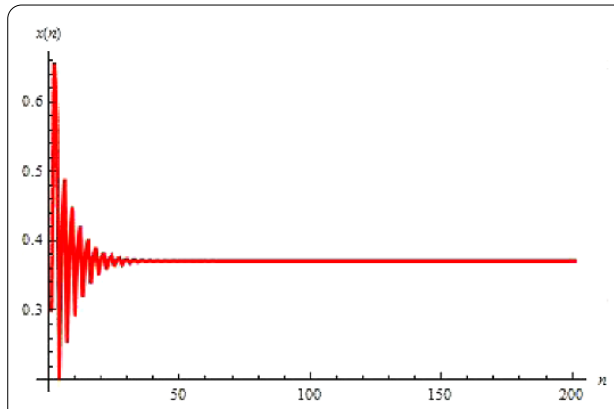

(a) Plot of $x_{n}$ for system (29)

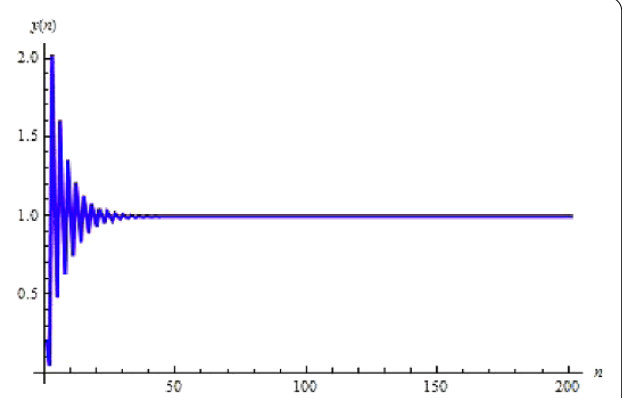

(b) Plot of $y_{n}$ for system (29)

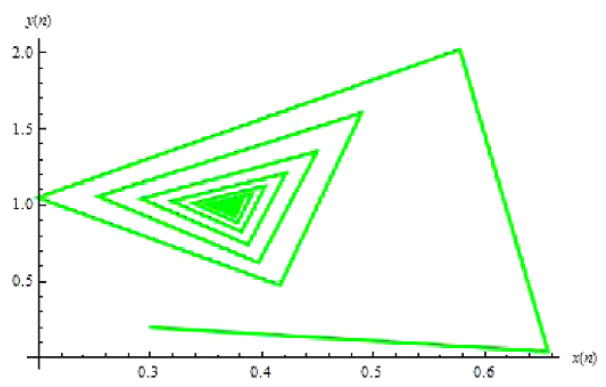

(c) An attractor of system (29)

Figure 5 Plots for system (29).

Example 6 Let $\alpha=1,245, \beta=111, \gamma=1,266, \alpha_{1}=1.1, \beta_{1}=32, \gamma_{1}=0.9$. Then system (2) can be written as

$$
x_{n+1}=\frac{1,245 e^{-x_{n}}+111 e^{-x_{n-1}}}{1,266+1,245 y_{n}+111 y_{n-1}}, \quad y_{n+1}=\frac{1.1 e^{-y_{n}}+32 e^{-y_{n-1}}}{0.9+1.1 x_{n}+32 x_{n-1}},
$$

with initial conditions $x_{-1}=111.7, x_{0}=0.3, y_{-1}=0.8, y_{0}=0.8$.

In this case the unique positive equilibrium point of system (30) is unstable. Moreover, in Figure 6 the plot of $x_{n}$ is shown in Figure 6(a), the plot of $y_{n}$ is shown in Figure 6(b), and a phase portrait of system (30) is shown in Figure 6(c).

Example 7 Let $\alpha=1,145, \beta=201, \gamma=1,266, \alpha_{1}=15, \beta_{1}=232, \gamma_{1}=3$. Then system (2) can be written as

$$
x_{n+1}=\frac{1,145 e^{-x_{n}}+201 e^{-x_{n-1}}}{1,266+1,145 y_{n}+201 y_{n-1}}, \quad y_{n+1}=\frac{15 e^{-y_{n}}+232 e^{-y_{n-1}}}{3+15 x_{n}+232 x_{n-1}},
$$

with initial conditions $x_{-1}=0.9, x_{0}=0.5, y_{-1}=0.001, y_{0}=0.8$.

In this case the unique positive equilibrium point of system (31) is unstable. Moreover, in Figure 7 the plot of $x_{n}$ is shown in Figure 7(a), the plot of $y_{n}$ is shown in Figure 7(b), and a phase portrait of system (31) is shown in Figure 7(c).

Example 8 Let $\alpha=2,145, \beta=166, \gamma=2,566, \alpha_{1}=16, \beta_{1}=252, \gamma_{1}=3$. Then system (2) can be written as

$$
x_{n+1}=\frac{2,145 e^{-x_{n}}+166 e^{-x_{n-1}}}{2,566+2,145 y_{n}+21 y_{n-1}}, \quad y_{n+1}=\frac{16 e^{-y_{n}}+252 e^{-y_{n-1}}}{3+16 x_{n}+252 x_{n-1}},
$$

with initial conditions $x_{-1}=2.9, x_{0}=0.3, y_{-1}=0.02, y_{0}=1.7$. 


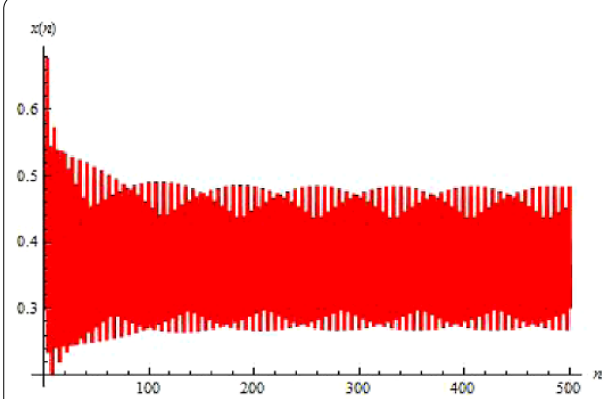

(a) Plot of $x_{n}$ for system (30)

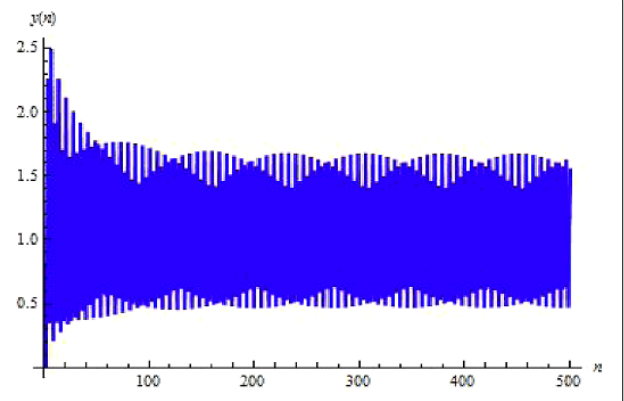

(b) Plot of $y_{n}$ for system (30)

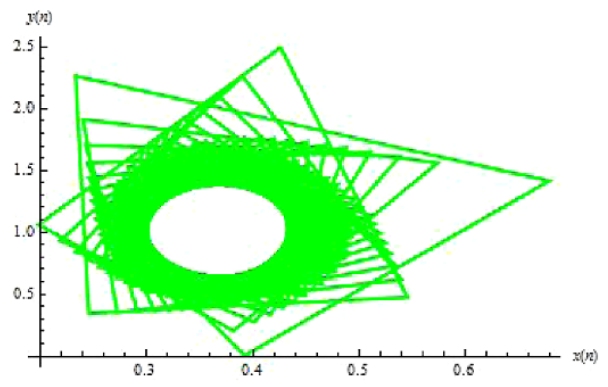

(c) Phase portrait of system (30)

Figure 6 Plots for system (30).

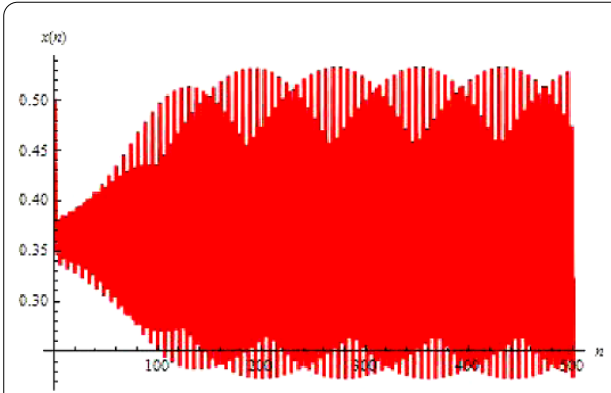

(a) Plot of $x_{n}$ for system (31)

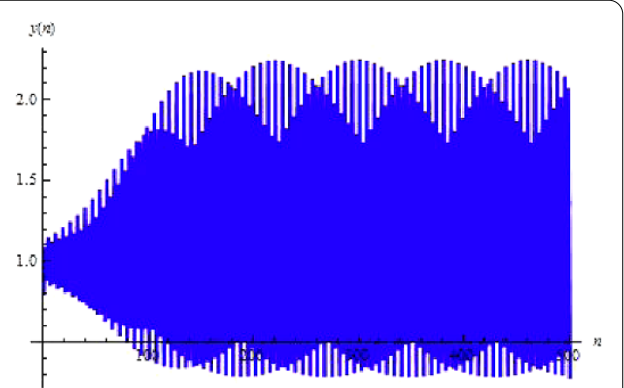

(b) Plot of $y_{n}$ for system (31)

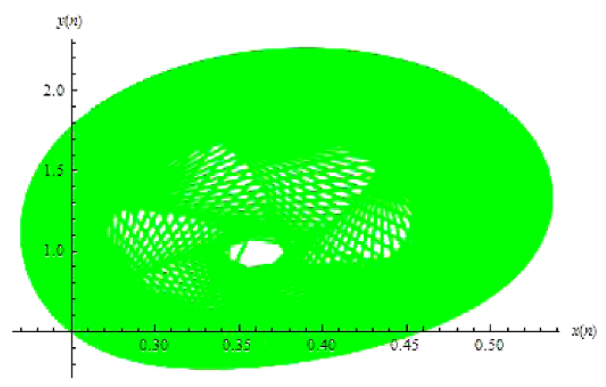

(c) Phase portrait of system (31)

Figure 7 Plots for system (31). 


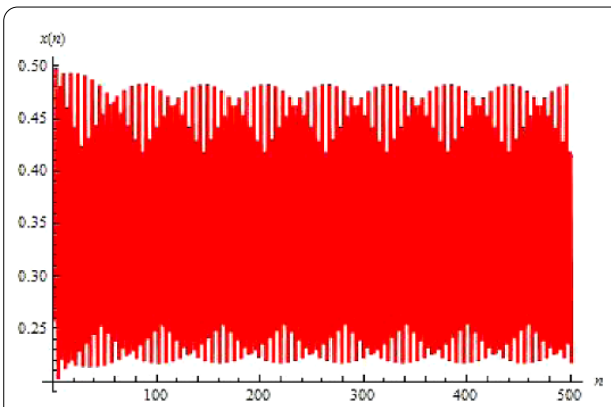

(a) Plot of $x_{n}$ for system (32)

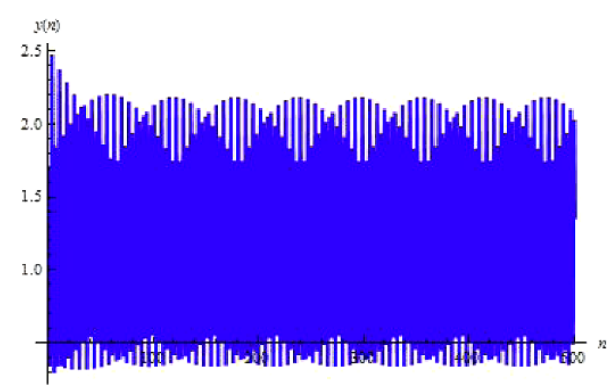

(b) Plot of $y_{n}$ for system (32)

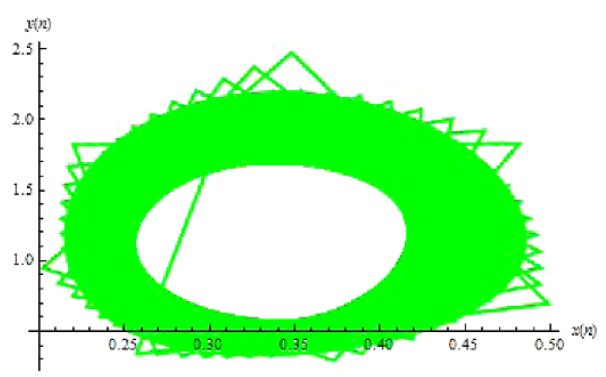

(c) Phase portrait of system (32)

Figure 8 Plots for system (32).

In this case the unique positive equilibrium point of system (32) is unstable. Moreover, in Figure 8 the plot of $x_{n}$ is shown in Figure 8(a), the plot of $y_{n}$ is shown in Figure 8(b), and a phase portrait of system (32) is shown in Figure 8(c).

\section{Conclusion}

This work is related to the qualitative behavior of some systems of exponential rational difference equations. We have investigated the existence and uniqueness of the positive steady state of system (1) and (2). For all positive values of the parameters the boundedness and persistence of positive solutions are proved. Moreover, we have shown that the unique positive equilibrium point of system (1) and (2) is locally as well as globally asymptotically stable under certain parametric conditions. The main objective of dynamical systems theory is to predict the global behavior of a system based on the knowledge of its present state. An approach to this problem consists of determining the possible global behaviors of the system and determining which parametric conditions lead to these long-term behaviors. By constructing a discrete Lyapunov function, we have obtained the global asymptotic stability of the positive equilibrium of (1) and (2). Finally, some illustrative examples are provided to support our theoretical discussion. First two examples show that the unique positive equilibrium point of system (1) is stable with different parametric values. Meanwhile Examples 3, 4, and 5 show that the unique positive equilibrium point of system (2) is stable whereas the last three examples show that the unique positive equilibrium point of system (2) is unstable with suitable parametric choices. 


\section{Acknowledgements}

The author thanks the main editor and anonymous referees for their valuable comments and suggestions leading to improvement of this paper. This work was supported by the Higher Education Commission of Pakistan.

Received: 25 September 2014 Accepted: 6 November 2014 Published: 26 Nov 2014

\section{References}

1. El-Metwally, E, Grove, EA, Ladas, G, Levins, R, Radin, M: On the difference equation $x_{n+1}=\alpha+\beta x_{n-1} e^{-x_{n}}$. Nonlinear Anal. 47, 4623-4634 (2001)

2. Ozturk, I, Bozkurt, F, Ozen, S: On the difference equation $y_{n+1}=\frac{\alpha+\beta e^{-y_{n}}}{\gamma+y_{n-1}}$. Appl. Math. Comput. 181, 1387-1393 (2006)

3. Bozkurt, F: Stability analysis of a nonlinear difference equation. Int. J. Mod. Nonlinear Theory Appl. 2, 1-6 (2013)

4. Papaschinopoulos, G, Radin, MA, Schinas, CJ: On the system of two difference equations of exponential form: $x_{n+1}=a+b x_{n-1} e^{-y_{n}}, y_{n+1}=c+d y_{n-1} e^{-x_{n}}$. Math. Comput. Model. 54, 2969-2977 (2011)

5. Papaschinopoulos, G, Radin, MA, Schinas, CJ: Study of the asymptotic behavior of the solutions of three systems of difference equations of exponential form. Appl. Math. Comput. 218, 5310-5318 (2012)

6. Papaschinopoulos, G, Schinas, CJ: On the dynamics of two exponential type systems of difference equations. Comput. Math. Appl. 64(7), 2326-2334 (2012)

7. Khan, AQ, Qureshi, MN: Behavior of an exponential system of difference equations. Discrete Dyn. Nat. Soc. 2014 Article ID 607281 (2014). doi:10.1155/2014/60728

8. Din, Q, Qureshi, MN, Khan, AQ: Dynamics of a fourth-order system of rational difference equations. Adv. Differ. Equ. 2012, Article ID 215 (2012)

9. Kulenović, MRS, Ladas, G: Dynamics of Second Order Rational Difference Equations. Chapman \& Hall/CRC, London (2002)

10. Elsayed, EM: Solutions of rational difference system of order two. Math. Comput. Model. 55, 378-384 (2012)

11. Elsayed, EM: Behavior and expression of the solutions of some rational difference equations. J. Comput. Anal. Appl. 15(1), 73-81 (2013)

12. Elsayed, EM, El-Metwally, H: Stability and solutions for rational recursive sequence of order three. J. Comput. Anal. Appl. 17(2), 305-315 (2014)

13. Elsayed, EM, El-Metwally, HA: On the solutions of some nonlinear systems of difference equations. Adv. Differ. Equ. 2013, Article ID 16 (2013)

14. Din, Q, Khan, AQ, Qureshi, MN: Qualitative behavior of a host-pathogen model. Adv. Differ. Equ. 2013, Article ID 263 (2013)

15. Khan, AQ, Qureshi, MN, Din, Q: Global dynamics of some systems of higher-order rational difference equations. Adv. Differ. Equ. 2013, Article ID 354 (2013)

16. Qureshi, MN, Khan, AQ, Din, Q: Asymptotic behavior of a Nicholson-Bailey model. Adv. Differ. Equ. 2014, Article ID 62 (2014)

17. Sedaghat, H: Nonlinear Difference Equations: Theory with Applications to Social Science Models. Kluwer Academic, Dordrecht (2003)

18. Pituk, M: More on Poincaré's and Perron's theorems for difference equations. J. Differ. Equ. Appl. 8, 201-216 (2002)

19. Enatsu, Y, Nakata, Y, Muroya, Y: Global stability for a class of discrete SIR epidemic models. Math. Biosci. Eng. 7(2), 347-361 (2010)

20. Kocic, VL, Ladas, G: Global Behavior of Nonlinear Difference Equations of Higher Order with Applications. Kluwer Academic, Dordrecht (1993)

10.1186/1687-1847-2014-297

Cite this article as: Khan: Global dynamics of two systems of exponential difference equations by Lyapunov function. Advances in Difference Equations 2014, 2014:297

\section{Submit your manuscript to a SpringerOpen ${ }^{\ominus}$ journal and benefit from:}

- Convenient online submission

Rigorous peer review

- Immediate publication on acceptance

- Open access: articles freely available online

- High visibility within the field

- Retaining the copyright to your article 